\title{
Health and functional status of tiotropium/olodaterol-treated patients with COPD: results from the AERIAL ${ }^{\circledR}$ non-interventional
} study

\author{
Adrian Gillissen ${ }^{1}$, Andrea Marseille ${ }^{2}$, Dirk Skowasch ${ }^{3}$, John Ritz $^{4}$, Muriel Mattiucci-Guehlke ${ }^{2}$, Stefan Pabst ${ }^{5}$, \\ Timm Greulich ${ }^{6,7}$ and Rembert Koczulla ${ }^{8}$
}

\begin{abstract}
${ }^{1}$ Medizinische Klinik III, Dept of Internal and Pulmonary Medicine, Klinikum am Steinenberg/Ermstalklinik, Reutlingen, Germany. ${ }^{2} \mathrm{HP}$ Country Medical Affairs, Boehringer Ingelheim Pharma GmbH \& Co. KG, Ingelheim am Rhein, Germany. ${ }^{3}$ Dept of Internal Medicine II Pneumology, University Hospital Bonn, Bonn, Germany. ${ }^{4}$ Biostatistics, Boehringer Ingelheim Pharma GmbH \& Co. KG, Ingelheim am Rhein, Germany. ${ }^{5}$ Lungenpraxis Bonn, Bonn, Germany. ${ }^{6}$ Dept of Medicine, Pulmonary and Critical Care Medicine, University Medical Center Giessen and Marburg, Member of the German Center for Lung Research, Marburg, Germany. ${ }^{7}$ PneumoPraxis-Marburg, Marburg, Germany. ${ }^{8}$ Institute for Pulmonary Rehabilitation Research, Schoen Clinic Berchtesgadener Land, Teaching Hospital of Philipps University of Marburg, Salzburg, Austria.
\end{abstract}

Corresponding author: Adrian Gillissen (gillissen_a@klin-rt.de)

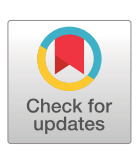

Copyright $\odot$ The authors 2021

This version is distributed under the terms of the Creative Commons Attribution NonCommercial Licence 4.0. For commercial reproduction rights and permissions contact permissions@ersnet.org

This article has supplementary material available from openres.ersjournals.com

Received: 4 Jan 2021 Accepted: 21 June 2021

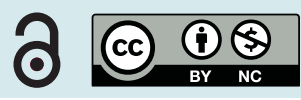

Shareable abstract (@ERSpublications)

The AERIAL non-interventional study demonstrated that patients with COPD treated with tiotropium/olodaterol via Respimat in routine clinical practice had high product satisfaction and clinically relevant improvements in health and functional status https://bit.ly/3jf6hlf

Cite this article as: Gillissen A, Marseille A, Skowasch D, et al. Health and functional status of tiotropium/olodaterol-treated patients with COPD: results from the AERIAL ${ }^{\circledR}$ non-interventional study. ERJ Open Res 2021; 7: 00004-2021 [DOI: 10.1183/23120541.00004-2021].

\section{Abstract}

Patients with COPD often have reduced physical activity, which can impair health status. Real-world data can provide valuable information on the health and functional status of patients with COPD treated with tiotropium/olodaterol.

AERIAL $^{\circledR}$ (ClinicalTrials.gov NCT03165045) was a German, non-interventional study of patients with COPD receiving treatment with tiotropium/olodaterol under real-world conditions for $\sim 6$ weeks. The primary end-point was the proportion of patients achieving a decrease of $\geqslant 0.4$ points in Clinical COPD Questionnaire (CCQ) score. The CCQ-4 subdomain was used to assess functional status, and the Physician's Global Evaluation (PGE) scale was used to assess the patients' general condition. Safety was assessed, as well as patient satisfaction and willingness to continue treatment.

Out of 1351 screened patients, 1322 were treated and 1140 comprised the full analysis set. The primary end-point was met: $66.3 \%$ of patients achieved a $\geqslant 0.4$-point decrease in overall CCQ score (mean \pm SD decrease 0.78 \pm 0.95$)$. Mean \pm SD decreases in CCQ symptoms and functional state subdomains were $0.84 \pm 1.06$ and $0.75 \pm 1.05$ points, respectively. PGE scores improved. One fatality (not treatment-related) and 23 drug-related adverse events were recorded, most commonly nausea and vertigo. $>85 \%$ of patients were satisfied/very satisfied with tiotropium/olodaterol overall and with the Respimat ${ }^{\circledR}$ device, both in terms of inhalation and handling. Most patients (95.2\%) expressed willingness to continue treatment.

Patients with COPD treated with tiotropium/olodaterol via Respimat ${ }^{\circledR}$ in routine clinical practice had clinically relevant improvements in health and functional status compared with baseline.

\section{Introduction}

COPD is a common and treatable disease that is characterised by airflow limitation and persistent respiratory symptoms [1]. Patients with COPD often have reduced physical activity [2, 3], which results in impaired health status and reduced health-related quality of life (HRQoL), and may contribute to hospitalisations and increased mortality [4-6].

Pharmacological therapy for COPD can reduce symptoms, decrease the frequency and severity of exacerbations, and improve health status and exercise tolerance [1]. Combination treatment with a 
long-acting muscarinic antagonist (LAMA) and long-acting $\beta_{2}$-agonist (LABA) increases forced expiratory volume in $1 \mathrm{~s}$ and reduces symptoms and exacerbations compared with monotherapy [1].

Tiotropium/olodaterol (Spiolto ${ }^{\circledR}$ ) is a fixed-dose LAMA/LABA combination, administered via a soft-mist inhaler (SMI; Respimat ${ }^{\circledR}$ Soft Mist ${ }^{\mathrm{TM}}$ inhaler), which has demonstrated greater improvements in lung function, symptoms, HRQoL and exercise capacity among patients with COPD than its monocomponents (tiotropium or olodaterol) or placebo in clinical studies [7-11]. Real-world evidence shows treatment effects in a broader patient population, as seen in routine clinical practice, thus providing valuable additional information.

At the time of planning this observational study, only one non-interventional study had reported on the health status and physical activity/functional status of patients with COPD treated with tiotropium/ olodaterol in routine clinical practice [12]. This study was the first to provide evidence of improved physical functioning, measured by the self-reported 10-item Physical Functioning Questionnaire (PF-10), which translated into an overall improvement in the general condition of patients with COPD receiving tiotropium and olodaterol (in separate inhalers) [12].

The current study (AERIAL ${ }^{\circledR}$; ClinicalTrials.gov registration number NCT03165045) was conducted to generate real-world data regarding the effect of tiotropium/olodaterol on the health and functional status of patients with COPD. The Clinical COPD Questionnaire (CCQ) was used to assess changes in the health and functional status of patients with COPD who were treated for $\sim 6$ weeks with tiotropium/olodaterol via the Respimat ${ }^{\circledR}$ SMI in routine clinical practice in Germany.

\section{Methods}

This was an open-label non-interventional study enrolling consenting patients with COPD, who received treatment with tiotropium/olodaterol according to the approved summary of product characteristics (SmPC) [13]. Patients were observed for $\sim 6$ weeks under real-world conditions. Site selection was performed to reflect routine COPD care in Germany and ensure representativeness of the COPD population; therefore, the study included physicians who routinely treat COPD patients in an outpatient setting. Sites were selected randomly via phonebook research (general practitioners and specialists alike), as well as on the basis of physicians participating in a prior non-interventional study, SPIRIT.

The feasibility form covered estimated number of patients in Global Initiative for Chronic Obstructive Lung Disease (GOLD) group B-D within the past 12 months, within the upcoming 12 months, and a question regarding conflicting study projects. To minimise patient selection bias, consecutive enrolment was employed.

European Commission approval was obtained for the study and the German Federal Institute for Drugs and Medical Devices was notified. The study has been registered with both the European Network of Centres for Pharmacoepidemiology and Pharmacovigilance and www.clinicaltrials.gov.

The study included patients meeting all of the following criteria: 1) written informed consent prior to participation; 2) aged $\geqslant 40$ years; 3) diagnosed with COPD and having an indication for treatment with a combination of two long-acting bronchodilators (LAMA/LABA) according to approved SmPC [13] and GOLD strategy report 2017 (GOLD COPD groups B-D) [14]; and 4) treatment with tiotropium/olodaterol according to SmPC at the discretion of the physician. Patients were excluded if any of the following conditions were met: 1) contraindications according to the tiotropium/olodaterol SmPC; 2) already on a LAMA/LABA combination (free or fixed dose) in the 6 weeks prior to study entry; 3) continuing LABA/ inhaled corticosteroid (ICS) treatment in parallel with tiotropium/olodaterol (to avoid double-dosing of LABA); 4) pregnant or lactating; or 5) current participation in any clinical trial or any other noninterventional study of a drug or device.

As per the statistical analysis plan, patients violating any inclusion or exclusion criterion were retained in all analyses (treated set and full analysis set) and listed accordingly in the final statistical report and in the final study report.

The primary end-point was the proportion of patients achieving "therapeutic success", pre-defined as a $\geqslant 0.4$-point decrease in the CCQ score between visit 1 (baseline visit) and visit 2 (final visit, 6 weeks after visit 1). The secondary end-points were 1) changes in total CCQ score and CCQ-4 (functional status domain) score from visit 1 to visit 2; 2) patients' general condition at visit 1 and 2; and 3) patient satisfaction and willingness to continue treatment with tiotropium/olodaterol at visit 2. The CCQ includes 
10 questions relating to symptoms, and functional and mental status. Each question was scored by the patients on a 7-point scale from 0 to 6; the sum of the scores divided by 10 provides the CCQ score, with a higher score indicative of worse status. Functional status (CCQ-4), calculated as the sum of questions $7-$ 10 divided by four, assesses limitations in moderate and strenuous physical, daily and social activities. A change of 0.4 points is considered to be the minimal clinically important difference for both the total CCQ and CCQ-4 score $[15,16]$.

The treating physician completed the Physician's Global Evaluation (PGE) to evaluate patients' general condition, on an 8-point ordinal scale (from 1 (very poor) to 8 (excellent)). A patient satisfaction survey was completed at visit 2, using a 7-point ordinal scale (very dissatisfied to very satisfied). Willingness to continue treatment was assessed by a yes/no question at visit 2 .

Subgroup analyses were performed to assess therapeutic success according to prior use of COPD maintenance therapy (LABA only, LAMA only, or LABA/ICS) and GOLD ABCD classification. Patients who had not received COPD maintenance therapy in the 6 weeks prior to the study were considered maintenance-naïve. Adverse drug reactions (serious and nonserious) and fatal adverse events were assessed; all adverse events occurring after signing of informed consent and up to visit 2 were considered treatment-emergent.

Data were collected from patients enrolled between March 2017 and November 2018. AERIAL ${ }^{\circledR}$ was completed in adherence with the International Conference on Harmonization good clinical practice guidelines, with ethical approval received from the state medical council of Baden-Württemberg, Germany.

\section{Sample size calculation}

A CCQ therapeutic success rate similar to the St George's Respiratory Questionnaire (SGRQ) responder rate was assumed. In the TONADO studies, patients with COPD treated with tiotropium/olodaterol had a 57.5\% SGRQ responder rate [7]. A more diverse, real-world population would probably show a lower value than the selected trial population in the TONADO studies. Therefore, a 50\% CCQ therapeutic success rate was considered a reasonable assumption. Assuming this and a sample size of 1170 patients, the $95 \%$ confidence interval for the therapeutic success rate would be between $47.1 \%$ (lower limit) and 52.9\% (upper limit).

Subgroups treated with long-acting bronchodilators at visit 1 (i.e. LABA only, LAMA only, LABA/ICS) were analysed only if they included $>20 \%$ of all patients. In the smallest subgroup (with $\geqslant 234$ patients), assuming a $50 \%$ therapeutic success rate, the $95 \%$ CI would be between $43.6 \%$ and $56.4 \%$. To account for a $10 \%$ dropout rate, the sample size was increased to 1300 patients.

\section{Statistical analysis}

Primary and secondary end-points were analysed on the full analysis set, defined as patients with at least one documented administration of tiotropium/olodaterol (this was documented in the patient file and information was taken over into the electronic case report form), available CCQ scores at both visits and written informed consent. Safety end-points and demographic/baseline data were analysed on the treated set, comprising all patients with at least one documented administration of tiotropium/olodaterol and written informed consent.

For the primary end-point, the percentage of patients with therapeutic success is presented together with the 95\% CI. For comparison of subgroups, Chi-squared test or Fisher's exact test (if Chi-squared test was not valid) was used and p-values were interpreted nominally. For the secondary end-points, all analyses were descriptive. Missing observations (i.e. missing values or missing subgroup outcomes) were not considered for comparisons. No formal hypothesis testing was performed due to the self-controlled nature of the study. Analyses were carried out using SAS $^{\circledR} 9.3$ software, and were performed by Alcedis GmbH, Germany.

\section{Results}

\section{Patient disposition and baseline characteristics}

A total of 1351 patients were recruited from 114 sites in Germany (figure 1). These included general and specialty practice sites (general medicine $n=28$; internal medicine $n=22$; pneumology $n=4$; internal medicine and pneumology $n=59$; internal medicine and general medicine $n=1$ ). 29 patients were excluded, resulting in 1322 patients in the treated set (figure 1). A further 182 patients had missing total CCQ scores at visit 1 and/or visit 2, resulting in a full analysis set comprising 1140 patients. 


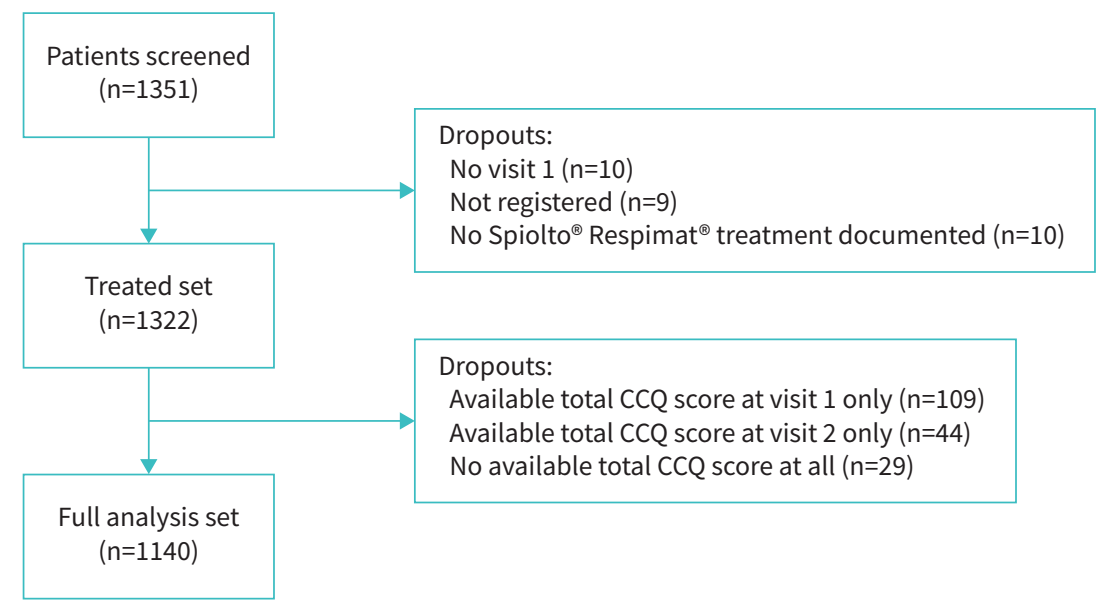

FIGURE 1 Patient disposition. Of the nine patients who were not registered, the reasons were violation of inclusion criterion $2(n=2)$, allocation to Global Initiative for Chronic Obstructive Lung Disease COPD group A $(n=3)$, meeting an exclusion criterion (unspecified, $n=1$ ), ongoing combined long-acting $\beta_{2}$-agonist (LABA) + inhaled corticosteroid therapy $(n=1)$ or LABA/long-acting muscarinic antagonist therapy $(n=2)$. There were no protocol violations due to informed consent. CCQ: Clinical COPD Questionnaire.

The median (range) age of patients in the treated set was 65 (40-104) years; 50.5\% of patients were aged $\leqslant 65$ years (table 1$)$. There were 760 (57.5\%) male patients; 612 (46.3\%) patients were current smokers, and 548 (41.5\%) were ex-smokers. 831 (62.9\%) patients were classified as GOLD group B, 321 (24.3\%) were classified as GOLD group D, $153(11.6 \%)$ as GOLD group C and one (0.1\%; a protocol violation) as GOLD group A (table 1). The majority of patients had either GOLD stage 2 (43.0\%) or GOLD stage 3 (38.1\%) airflow limitation; $48.0 \%$ of patients had grade 2 breathlessness, as per the modified UK Medical Research Council questionnaire. More than half (61.3\%) of patients had at least one exacerbation within the previous 12 months, and $12.2 \%$ had at least one exacerbation leading to hospitalisation during this period (table 1). Concomitant diseases were reported in 923 (69.8\%) patients, the most frequent of which was cardiac disease (48.0\%).

In the 6 weeks before the study, 258 (19.5\%) patients were treated with short-acting $\beta_{2}$-agonists, 228 (17.3\%) with a LAMA, 131 (9.9\%) with a LABA, and 45 (3.4\%) with ICS only. More than half (n=718, $54.3 \%$ ) of the patients had no COPD treatment in the 6 weeks before the study (table 1). Most patients (98.4\%) were trained on how to correctly inhale tiotropium/olodaterol before treatment started.

From the full analysis set population, 959 patients (84.1\%) were maintenance-naïve at study enrolment. The mean total CCQ score at visit 1 was 3.12 (mean symptom score 3.42; mean functional state score 3.05; mean mental state score 2.69).

\section{Efficacy}

Therapeutic success was achieved in 66.3\% of patients (95\% CI 63.49-69.06) (figure 2). When stratified by prior COPD maintenance therapy, a higher proportion of patients who were previously maintenance-naïve achieved therapeutic success (68.5\%) compared with those who were pre-treated with LABA only, LAMA only or LABA/ICS (54.7\%; figure 2). When stratified by GOLD groups, the proportion of patients with therapeutic success was highest in GOLD group D (75.5\%), followed by C (74.2\%) and B (61.5\%) (figure 2). When stratified by exacerbation history, the proportion of patients with therapeutic success increased with increasing exacerbation history (exacerbations in prior year: 0 (therapeutic success $56.8 \%) ; 1(67.4 \%) ; \geqslant 2(77.1 \%)$ ) (supplementary table 1$)$. The proportion of patients with therapeutic success stratified by exacerbation frequency, GOLD spirometric classification and baseline ICS use is provided in supplementary tables 1-3.

The mean \pm SD absolute change in CCQ total score from visit 1 to visit 2 was $0.78 \pm 0.95$ (figure 3 ). Regarding the CCQ subdomains, the largest mean absolute changes were observed in the symptoms subdomain $(0.84 \pm 1.06)$ and functional state subdomain $(0.75 \pm 1.05)$ (figure 3 ). Patients who were maintenance-naïve before study enrolment achieved greater absolute changes in total CCQ score, and in 


\begin{tabular}{|c|c|}
\hline Total number of patients & $1322(100.00)$ \\
\hline Age $\leqslant 65$ years & $668(50.53)$ \\
\hline Male & $760(57.49)$ \\
\hline \multicolumn{2}{|l|}{ Smoking status } \\
\hline Current smoker & $612(46.29)$ \\
\hline Ex-smoker & $548(41.45)$ \\
\hline Never-smoker & $162(12.25)$ \\
\hline \multicolumn{2}{|l|}{ COPD severity (GOLD groups) } \\
\hline A & $1(0.08)$ \\
\hline B & $831(62.86)$ \\
\hline C & $153(11.57)$ \\
\hline $\mathrm{D}$ & $321(24.28)$ \\
\hline Missing & $16(1.21)$ \\
\hline \multicolumn{2}{|l|}{ GOLD spirometric classification } \\
\hline 1 & $29(2.19)$ \\
\hline 2 & $569(43.04)$ \\
\hline 3 & $503(38.05)$ \\
\hline 4 & $134(10.14)$ \\
\hline No preliminary examination result & $87(6.58)$ \\
\hline \multicolumn{2}{|l|}{ mMRC questionnaire classification } \\
\hline Grade 0 & $51(3.86)$ \\
\hline Grade 1 & $103(7.79)$ \\
\hline Grade 2 & $635(48.03)$ \\
\hline Grade 3 & $279(21.10)$ \\
\hline Grade 4 & $238(18.00)$ \\
\hline Missing & $16(1.21)$ \\
\hline \multicolumn{2}{|l|}{ Exacerbations in the past 12 months } \\
\hline 0 & $512(38.73)$ \\
\hline 1 & $405(30.64)$ \\
\hline$\geqslant 2$ & $405(30.64)$ \\
\hline$\geqslant 1$ exacerbation leading to hospitalisation & $161(12.18)$ \\
\hline Patients with concomitant diseases & $923(69.82)$ \\
\hline Cardiac disease & $634(47.96)$ \\
\hline Allergic disease & $17(1.29)$ \\
\hline \multicolumn{2}{|l|}{ Patient trained to use Respimat inhaler } \\
\hline No & $21(1.59)$ \\
\hline Yes & $1301(98.41)$ \\
\hline \multicolumn{2}{|c|}{ Pharmacologically treated for COPD in the 6 weeks prior to start of study treatment } \\
\hline No & $718(54.31)$ \\
\hline Yes & $604(45.69)$ \\
\hline \multicolumn{2}{|l|}{ Type of treatment for COPD in the 6 weeks prior to start of study treatment } \\
\hline Short-acting $\beta_{2}$-agonist & $258(19.52)$ \\
\hline LABA & $131(9.91)$ \\
\hline Short-acting anticholinergic & $9(0.68)$ \\
\hline Long-acting anticholinergic & $228(17.25)$ \\
\hline Long-acting anticholinergic+LABA in fixed combination & $6(0.45)$ \\
\hline Short-acting anticholinergic+short-acting $\beta_{2}$-agonist in fixed combination & $69(5.22)$ \\
\hline$L A B A+I^{C} S^{\#}$ & $49(3.71)$ \\
\hline ICS & $45(3.40)$ \\
\hline Systemic corticosteroid & $5(0.38)$ \\
\hline Theophylline & $6(0.45)$ \\
\hline Roflumilast & $12(0.91)$ \\
\hline Other & $5(0.38)$ \\
\hline Missing & $2(0.15)$ \\
\hline
\end{tabular}

Data are presented as n (\%). GOLD: Global Initiative for Chronic Obstructive Lung Disease; mMRC: modified UK Medical Research Council; LABA: long-acting $\beta_{2}$-agonist; ICS: inhaled corticosteroid. \#: none of the 49 patients who were on LABA+ICS prior to the study continued ICS treatment or received a new prescription for ICS treatment during the study.

symptoms and functional state subdomain scores, compared with those who were pre-treated (supplementary table 4). Patients in GOLD groups C and D achieved greater absolute changes in total CCQ and subdomain scores compared with patients in GOLD group B (supplementary table 4). 


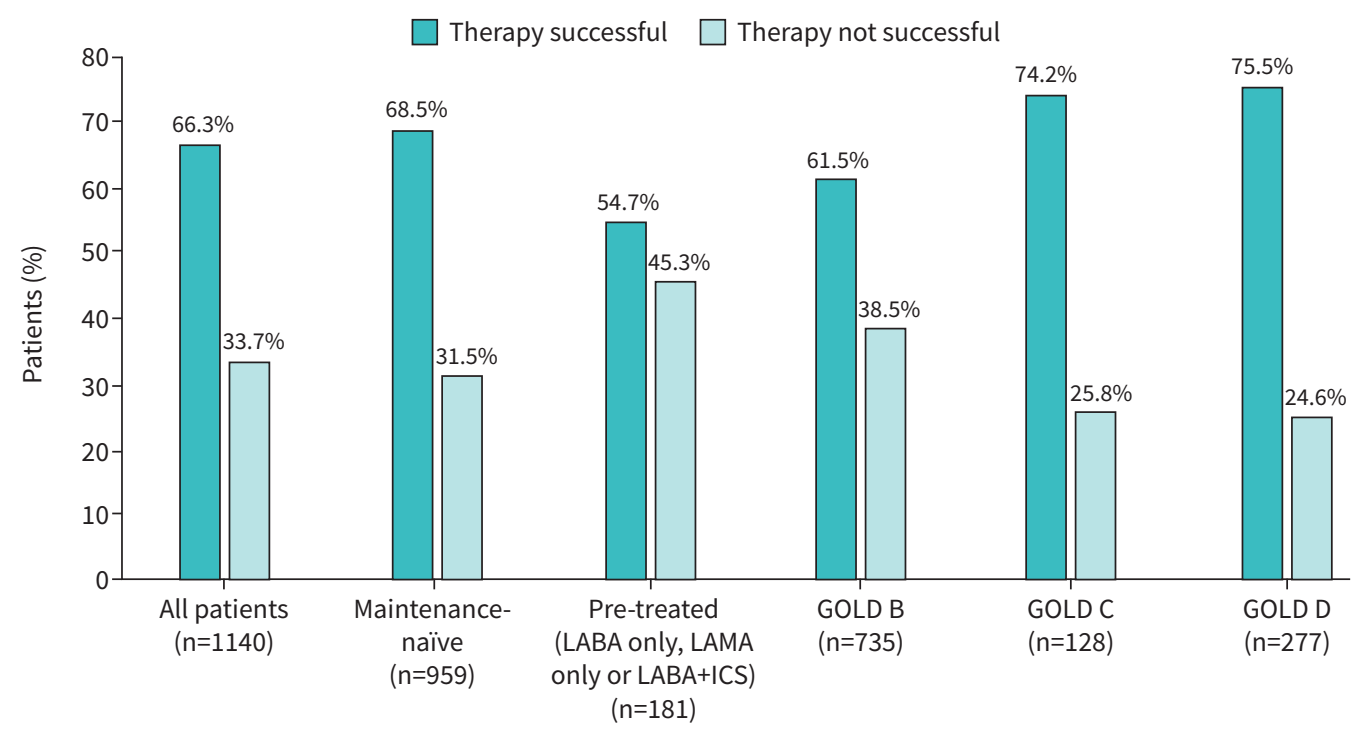

FIGURE 2 Proportion of patients achieving therapeutic success at visit 2. Therapeutic success was defined as a 0.4-point decrease in Clinical COPD Questionnaire score between visit 1 and visit 2. LABA: long-acting $\beta_{2}$-agonist; LAMA: long-acting muscarinic antagonist; ICS: inhaled corticosteroid; GOLD: Global Initiative for Chronic Obstructive Lung Disease.

Patients' general condition improved during the treatment period. At visit 1, most patients had a PGE score of $4(n=378,33.2 \%)$, followed by those with scores of $3(n=281,24.7 \%)$ and $5(n=195,17.1 \%)$ (where PGE score 1-2 is poor, 3-4 is satisfactory, 5-6 is good, and 7-8 is excellent). At visit 2, most patients had a PGE score of $5(n=350,33.7 \%)$, followed by those with scores of $6(n=325,28.5 \%)$ and $4(n=223$, 19.6\%) (figure 4). PGE scores were significantly lower in maintenance-naïve patients than in pre-treated patients at visit 1; however, there was no statistically significant difference between the groups at visit 2; improvements in PGE scores were observed in both groups from visit 1 to visit 2 . Stratification according to GOLD groups revealed that GOLD group D patients had lower PGE scores at both study visits (but also improved from visit 1 to visit 2).

Regarding patient satisfaction, 971 (85.2\%) patients were "very satisfied” or "satisfied” with tiotropium/ olodaterol overall; when asked specifically about inhalation and handling, 1008 (88.4\%) and 979 (85.9\%) of patients were "very satisfied" or "satisfied", respectively, with the Respimat ${ }^{\circledR}$ device (figure 5). Of the

Visit $1 \square$ Visit 2

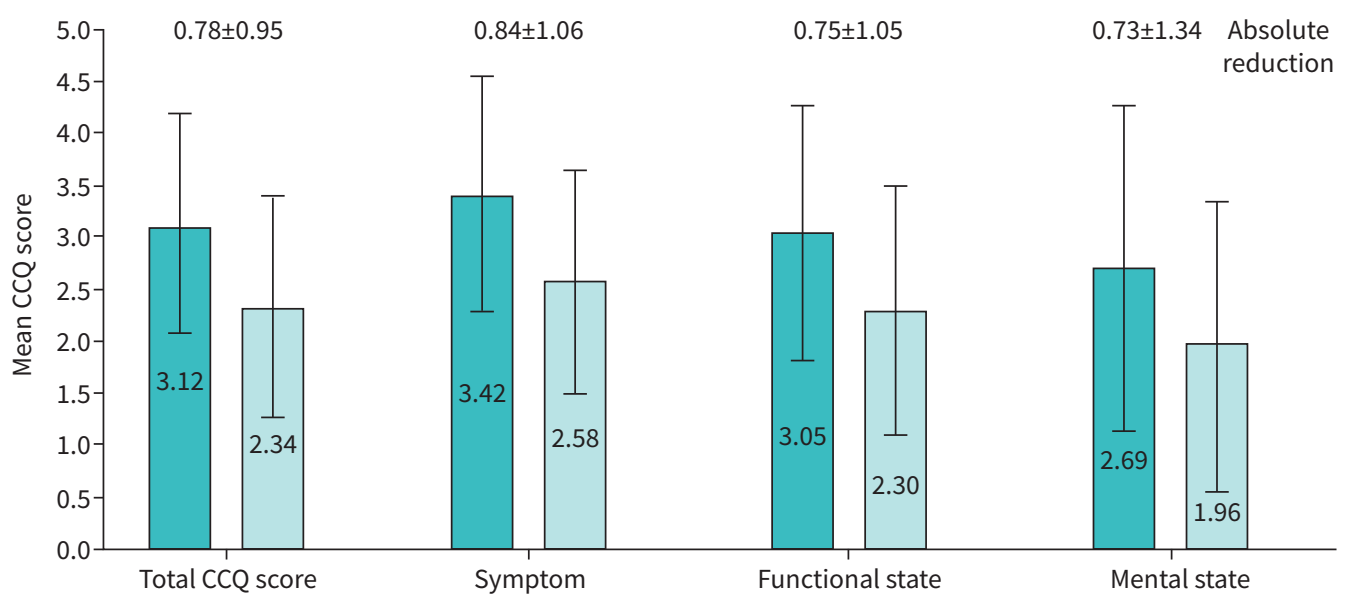

FIGURE 3 Absolute change in Clinical COPD Questionnaire (CCQ) score from visit 1 to visit 2. Data are presented as mean \pm sD. 


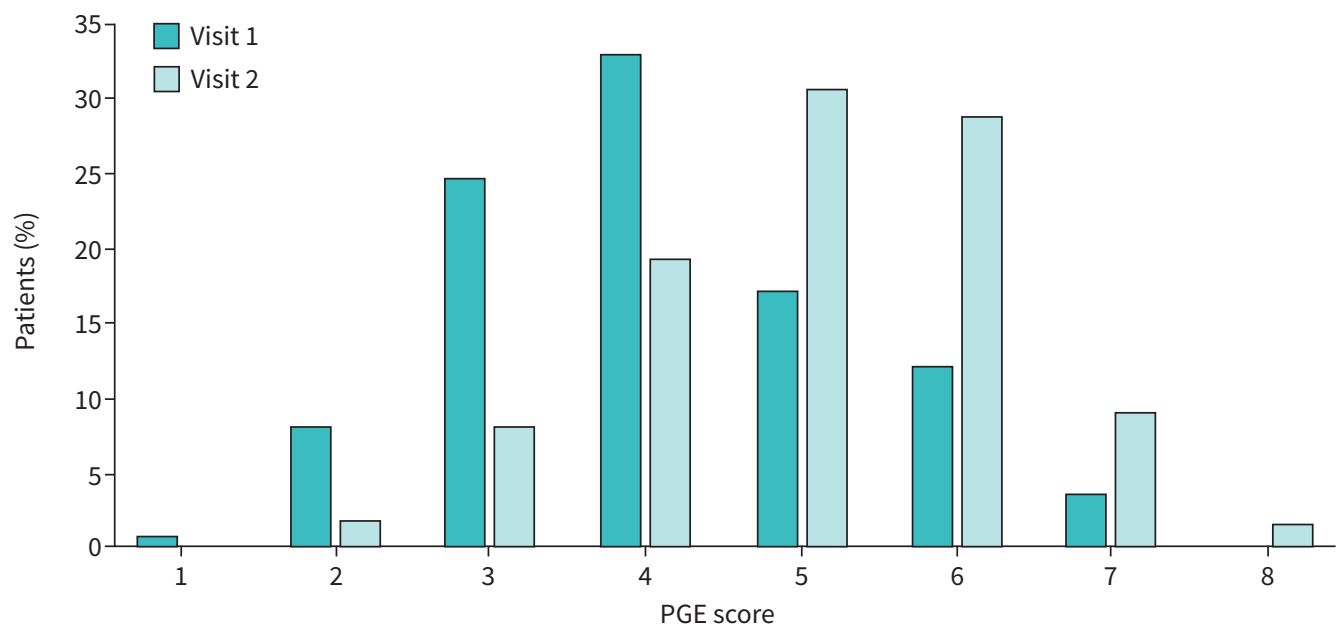

FIGURE 4 Patients' general condition (Physician's Global Evaluation (PGE) score) at visits 1 and 2. PGE score 12=poor, 3-4=satisfactory, 5-6=good, 7-8=excellent.

1254 patients attending visit 2, 1194 (95.2\%) expressed willingness to continue tiotropium/olodaterol treatment. Among the remaining 60 (4.8\%) patients, the most frequent reasons for therapy discontinuation were “patient’s wish” (n=38, 3\%) and “adverse events” ( $n=13,1 \%)$ (table 2).

\section{Requirement for additional medication}

Changes of COPD therapy were observed in 27 (2.2\%) patients between visits 1 and 2. For example, patients received additional short-acting $\beta_{2}$-agonists $(n=8,0.6 \%)$, ICS alone $(n=4,0.3 \%)$ and LABA, alone or in combination with a LAMA or ICS $(\mathrm{n}=8,0.6 \%)$.

\section{Safety}

Overall, 17 (1.3\%) patients experienced at least one investigator-defined drug-related adverse event, which led to treatment discontinuation in $13(1.0 \%)$ patients. None of the drug-related adverse events were serious. In total, 23 drug-related adverse events were documented. The most frequent of these were vertigo and nausea (three events each; $13 \%$ of all drug-related adverse events each), followed by dry mouth, peripheral oedema, paraesthesia and hypertension (two events each; 8.7\% of all drug-related adverse events each).

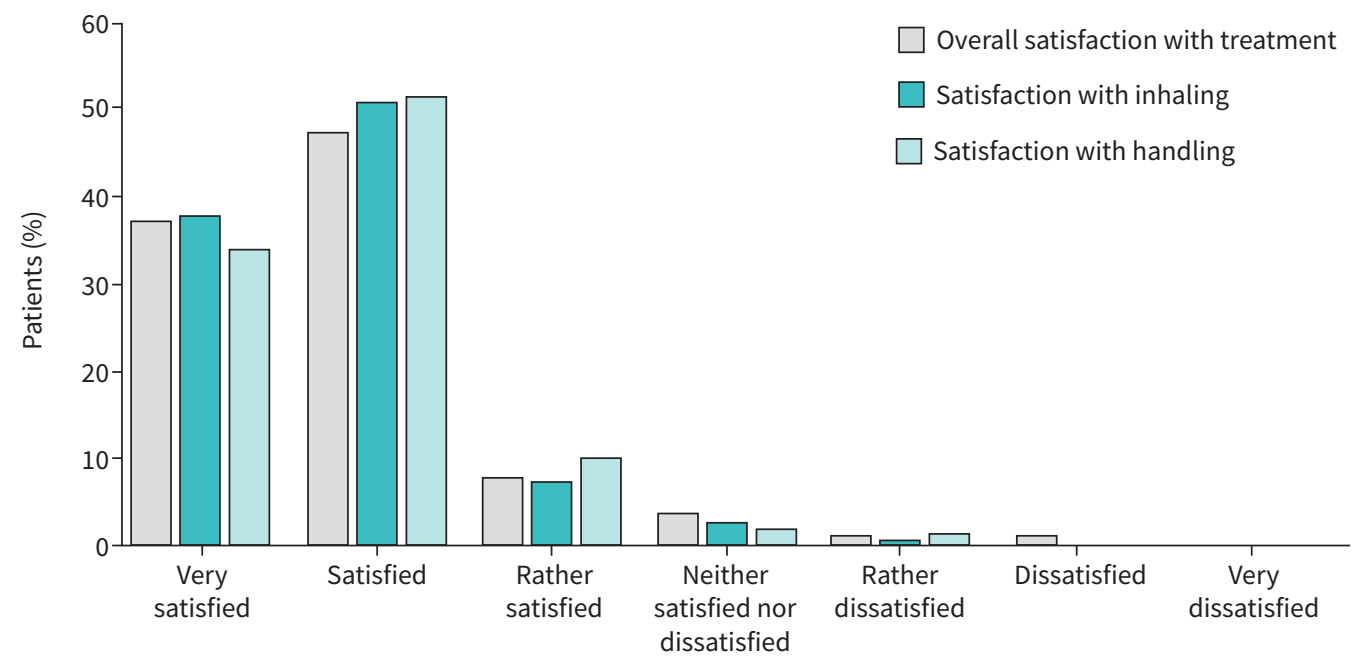

FIGURE 5 Patient satisfaction with tiotropium/olodaterol treatment overall, and in terms of inhaling from, and handling of the device at visit 2 . Visit 2 was $\sim 6$ weeks after visit 1 (baseline). 


\begin{tabular}{|c|c|}
\hline Patients with data at visit 2 & $1254(100.00)$ \\
\hline \multicolumn{2}{|c|}{ Patients willing to continue treatment } \\
\hline Yes & $1194(95.22)$ \\
\hline No & $60(4.78)$ \\
\hline \multicolumn{2}{|c|}{ Reason for treatment discontinuation } \\
\hline Adverse events & $13(1.04)$ \\
\hline Patient's wish & $38(3.03)$ \\
\hline Loss of contact & $1(0.08)$ \\
\hline Other & $8(0.64)$ \\
\hline
\end{tabular}

Only one serious adverse event (pulmonary embolism, one patient $(0.1 \%$ of all patients from the treated set)) was documented during the study, which led to hospitalisation and ultimately death. As assessed by the treating physician, there was no causal relationship between the event and study treatment.

\section{Discussion}

AERIAL ${ }^{\circledR}$ was a non-interventional study assessing changes in the health and functional status of patients with COPD treated with tiotropium/olodaterol for $\sim 6$ weeks in routine clinical practice. Therapeutic success was achieved by the majority of patients (66.3\%), so the primary end-point of the study was met. The mean total and subdomain CCQ scores decreased during the study, with greatest improvements seen in the symptom subdomain. Mean reductions in both the total and subdomain CCQ scores exceeded the minimal clinically important difference of 0.4 points, thus demonstrating clinical relevance.

A larger proportion of patients who were maintenance-naïve prior to the study achieved therapeutic success compared with patients who were pre-treated. In addition, maintenance-naïve patients showed larger absolute changes in total and subdomain CCQ scores (except for the mental state subdomain) versus patients who were pre-treated. This may be attributable to a greater capacity to benefit from bronchodilator therapy in patients not previously receiving maintenance COPD therapy compared with those who had already been treated. Other studies indicate that initiation of dual bronchodilation from the start of maintenance treatment onwards may result in greater improvements in lung function [17, 18], health status [18], dyspnoea severity [18] and use of rescue medication [17], as well as a reduced risk of short-term clinical deterioration [17], when compared with a single bronchodilator. However, this is not in line with the GOLD 2021 strategy report, which recommends dual bronchodilation with LAMA/LABA as initial therapy only for GOLD D patients with more severe symptoms (COPD Assessment Test ${ }^{\mathrm{TM}}$ score $\geqslant 20$ ) and GOLD $B$ patients with severe breathlessness [1]. In contrast, recent guidelines from the American Thoracic Society and the National Institute for Health and Care Excellence recommend the use of LAMA/LABA as first-line therapy in a wider range of patients $[19,20]$.

Of note, $84.1 \%$ of patients in this study were maintenance-naïve. This study included GOLD groups B, C and D patients, who, according to the GOLD 2021 strategy report, should be treated with a long-acting bronchodilator at a minimum [1]. This indicates a high rate of undertreatment and a lack of guideline adherence in real-world practice, which is consistent with other studies [21, 22]. More patients in GOLD groups C and D achieved therapeutic success than patients in GOLD group B, supporting the results of another observational study with tiotropium/olodaterol, which evaluated therapeutic success using PF-10 score [23].

PGE scores improved in all patients over the period assessed; improvements were larger in maintenance-naïve patients than in patients who were pre-treated, in line with the CCQ results. The improvements in PGE scores observed in our study provide further support for the benefits of treating symptomatic patients with LAMA/LABA therapy in accordance with the GOLD recommendations [1]. However, it is important to note that our study was not designed to analyse the superiority of tiotropium/ olodaterol in maintenance-naïve versus pre-treated patients.

Improvements in PGE scores were also seen in three other non-interventional studies with tiotropium/olodaterol, supporting the finding that tiotropium/olodaterol therapy improves patients' general condition [12, 23, 24].

Most patients were very satisfied or satisfied with tiotropium/olodaterol treatment overall, as well as with inhaling from and handling the Respimat ${ }^{\circledR}$ device. Only 2\% of patients expressed dissatisfaction. Patient satisfaction with inhalers and treatment is an important patient-reported outcome and can impact patient 
treatment adherence [25]. $>95 \%$ of patients reported willingness to continue tiotropium/olodaterol treatment beyond the study, although dropouts may have introduced bias here.

The results of this study are similar to those from two recently published open-label non-interventional studies evaluating COPD patients who received tiotropium/olodaterol for $\sim 6$ weeks in Germany. Treatment with tiotropium/olodaterol led to therapeutic success (defined as a 10-point increase in PF-10 score) in $\sim 50 \%$ of patients, and to improvements in the patients' general condition; moreover, the majority of patients were very satisfied or satisfied with the Respimat ${ }^{\circledR}$ inhaler [12, 23]. In contrast to these studies, the current study used the CCQ to measure health and functional status. This is a strength of the AERIAL ${ }^{\circledR}$ study since the CCQ was developed and validated especially for COPD patients and is widely used to monitor COPD health status [26-28]. In addition, unlike the PF-10, the CCQ contains separate domains for symptoms and mental and functional state [15]. In terms of patient population, the AERIAL ${ }^{\circledR}$ study was similar to the studies by STEINMETZ et al. [23] and SAUER et al. [12] with respect to the proportion of males (57\% and 59\%, respectively), current smokers (40\% and $35 \%$, respectively) and prevalence of cardiac disease (51\% and 53\%, respectively). However, distribution of GOLD ABCD groups varied, with a predominance of GOLD A patients in the study by STEINMETz et al. [23] (42\%) compared with 0.1\% of patients in the AERIAL ${ }^{\circledR}$ study and $0 \%$ in the study by SAUER et al. [12]; the AERIAL ${ }^{\circledR}$ study had the highest proportion of GOLD B patients. The proportion of treatment-naïve patients also varied between studies and was highest in the AERIAL ${ }^{\circledR}$ study (54\% (with $84 \%$ maintenance-naïve) versus $31 \%$ and $46 \%$ in the SAuer et al. [12] and Steinmetz et al. [23] studies, respectively). The proportion of patients receiving LABA/ICS prior to study entry was lower in our study (3.7\% versus 9-21\%) compared with other observational studies evaluating tiotropium/olodaterol [12, 23, 29]. Similarly, the DACCORD study, a non-interventional study recruiting patients following COPD maintenance treatment change or initiation in Germany enrolled a greater proportion of patients on LABA/ICS at baseline (22\%) [30]. The lower proportion of patients in our study may be due to compliance with GOLD 2017 recommendations, which advise prescribing LABA/ICS to only some patients with GOLD group D COPD [14]. Hence, this low number may reflect guideline-recommended patient management, which might be more apparent in our study due to differences in recruitment compared with the other studies.

The number of patients with drug-related adverse events in the AERIAL ${ }^{\circledR}$ study (1.3\%) was lower than those observed in clinical trials $[7,10]$. This may be because drug-related adverse events in clinical trials are more actively screened by investigators because all adverse events have to be documented; the investigators are therefore more aware of adverse events in general than in an observational study, in which "only" drug-related adverse events (and fatal adverse events) need to be recorded. The reported drug-related adverse events are mostly in line with the adverse reactions listed in the SmPC for tiotropium/ olodaterol [13].

This study had some limitations. The duration ( 6 weeks) was short, preventing an assessment of long-term therapeutic effects. However, this duration corresponds to the usual treatment period before assessing the efficacy of a new COPD treatment. This was a non-interventional study and therefore did not include a comparator group; hence, data focusing on subjective parameters have to be considered cautiously. In addition, the non-interventional nature of the study prohibited monitoring of patient treatment adherence using a medication diary. In some subgroup analyses, there were imbalances in the number of patients in the subgroups. For example, there were considerably more maintenance-naïve than pre-treated patients (959 versus 181 patients) and more patients in GOLD group B versus GOLD groups C and D (735 versus 128 and 277, respectively). Therefore, the results of these subanalyses should be interpreted with caution. Another limitation was that the survey used to assess patient satisfaction at visit 2 was not a validated instrument.

This study has several strengths. Firstly, the study was performed under routine medical practice conditions, and therefore results can be transferred to a more heterogeneous patient population with COPD in real-world clinical settings. For example, $48 \%$ of patients in AERIAL ${ }^{\circledR}$ had cardiac disease at baseline, compared with a lower proportion in randomised clinical trials (RCTs) of tiotropium/olodaterol (e.g. 29\% had a cardiovascular history in a pooled analysis of TONADO 1 and 2 and DYNAGITO [31]). There was a lower proportion of males in our study (57\%) compared with the pooled analysis of tiotropium/olodaterol RCTs (71\%) and a higher proportion of current smokers (46\% versus 37\%); however, the average ages of the populations were similar (65-66 years) [31]. Secondly, since a great number of sites throughout Germany enrolled patients, we consider the results to be representative of COPD patients in this country.

The AERIAL ${ }^{\circledR}$ non-interventional study provides further real-world evidence to support the use of tiotropium/olodaterol via Respimat ${ }^{\circledR}$ in patients with COPD. Treatment with tiotropium/olodaterol under 
routine medical care conditions was effective and well tolerated, even in a patient population suffering from a variety of concomitant diseases, including almost half with cardiac disease.

\section{Conclusion}

Patients with COPD who were treated with tiotropium/olodaterol via Respimat $^{\circledR}$ in routine clinical practice in Germany had clinically relevant improvements in health status and functional status compared with baseline. The majority of patients participating in the study were very satisfied or satisfied with the drug treatment and device.

Acknowledgements: Medical writing assistance was provided by Vicki Cronin (MediTech Media, Manchester, UK), which was funded by Boehringer Ingelheim Pharma GmbH \& Co. KG.

Provenance: Submitted article, peer reviewed.

This study is registered at www.clinicaltrials.gov with identifier number NCT03165045.

Conflict of interest: A. Gillissen reports nonfinancial support from Boehringer Ingelheim during the conduct of the study. A. Marseille is an employee of Boehringer Ingelheim. D. Skowasch reports personal fees from Boehringer Ingelheim, AstraZeneca, Berlin-Chemie, GSK, Grifols and Novartis, outside the submitted work. J. Ritz is an employee of Boehringer Ingelheim. M. Mattiucci-Guehlke is an employee of Boehringer Ingelheim. S. Pabst has nothing to disclose. T. Greulich reports personal fees from AstraZeneca, Berlin-Chemie, Boehringer Ingelheim, GSK, Novartis, Roche and Chiesi, and grants and personal fees from CSL Behring and Grifols, outside the submitted work. R. Koczulla has nothing to disclose.

Support statement: This study was funded by Boehringer Ingelheim Pharma GmbH \& Co. KG. Funding information for this article has been deposited with the Crossref Funder Registry.

\section{References}

1 Global Initiative for Chronic Obstructive Lung Disease. Global Strategy for the Diagnosis, Management, and Prevention of Chronic Obstructive Pulmonary Disease (2021 report). https://goldcopd.org/wp-content/ uploads/2020/11/GOLD-REPORT-2021-v1.1-25Nov20_WMV.pdf Date last accessed: 2 February 2021.

2 Troosters T, Sciurba F, Battaglia S, et al. Physical inactivity in patients with COPD, a controlled multi-center pilot-study. Respir Med 2010; 104: 1005-1011.

3 Watz H, Waschki B, Meyer T, et al. Physical activity in patients with COPD. Eur Respir J 2009; 33: 262-272.

4 Esteban C, Quintana JM, Aburto M, et al. Impact of changes in physical activity on health-related quality of life among patients with COPD. Eur Respir J 2010; 36: 292-300.

5 Garcia-Aymerich J, Lange $\mathrm{P}$, Benet $\mathrm{M}$, et al. Regular physical activity reduces hospital admission and mortality in chronic obstructive pulmonary disease: a population based cohort study. Thorax 2006; 61: 772-778.

6 Almagro P, Calbo E, Ochoa de Echagüen A, et al. Mortality after hospitalization for COPD. Chest 2002; 121: 1441-1448.

7 Buhl R, Maltais F, Abrahams R, et al. Tiotropium and olodaterol fixed-dose combination versus mono-components in COPD (GOLD 2-4). Eur Respir J 2015; 45: 969-979.

8 O'Donnell DE, Casaburi R, Frith P, et al. Effects of combined tiotropium/olodaterol on inspiratory capacity and exercise endurance in COPD. Eur Respir J 2017; 49: 1601348.

9 Singh D, Gaga M, Schmidt O, et al. Effects of tiotropium+olodaterol versus tiotropium or placebo by COPD disease severity and previous treatment history in the OTEMTO ${ }^{\circledR}$ studies. Respir Res 2016; 17: 73.

10 Beeh KM, Westerman J, Kirsten AM, et al. The 24-h lung-function profile of once-daily tiotropium and olodaterol fixed-dose combination in chronic obstructive pulmonary disease. Pulm Pharmacol Ther 2015; 32: 53-59.

11 Maltais F, O'Donnell D, Gáldiz Iturri JB, et al. Effect of 12 weeks of once-daily tiotropium/olodaterol on exercise endurance during constant work-rate cycling and endurance shuttle walking in chronic obstructive pulmonary disease. Ther Adv Respir Dis 2018; 12: 1753465818755091.

12 Sauer R, Hänsel M, Buhl R, et al. Impact of tiotropium+olodaterol on physical functioning in COPD: results of an open-label observational study. Int J Chron Obstruct Pulmon Dis 2016; 11: 891-898.

13 Boehringer Ingelheim Limited. Spiolto Respimat 2.5 microgram/2.5 microgram, inhalation solution. Summary of Product Characteristics. www.medicines.org.uk/emc/medicine/30495 Date last accessed: 6 November 2019. Date last updated: 14 January 2021. 

Prevention of Chronic Obstructive Pulmonary Disease. http://goldcopd.org/gold-2017-global-strategy-diagnosismanagement-prevention-copd/ Date last accessed: 23 February 2018.

15 Kocks JW, Tuinenga MG, Uil SM, et al. Health status measurement in COPD: the minimal clinically important difference of the clinical COPD questionnaire. Respir Res 2006; 7: 62.

16 Kocks JWH, van de Ven M, Uil SM, et al. Functional status measurement in chronic obstructive pulmonary disease: the value of the functional status domain of the Clinical COPD Questionnaire. Thorax 2006; 61: S111.

17 Maleki-Yazdi MR, Singh D, Anzueto A, et al. Assessing short-term deterioration in maintenance-naïve patients with COPD receiving umeclidinium/vilanterol and tiotropium: a pooled analysis of three randomized trials. Adv Ther 2017; 33: 2188-2199.

18 Buhl R, de la Hoz A, Xue W, et al. Efficacy of tiotropium/olodaterol compared with tiotropium as a first-line maintenance treatment in patients with COPD who are naïve to LAMA, LABA and ICS: pooled analysis of four clinical trials. Adv Ther 2020; 37: 4175-4189.

19 Nici L, Mammen MJ, Charbek E, et al. Pharmacologic management of chronic obstructive pulmonary disease. An official American Thoracic Society clinical practice guideline. Am J Respir Crit Care Med 2020; 201: e56-e69.

20 National Institute for Health and Care Excellence. Chronic Obstructive Pulmonary Disease in Over 16s: Diagnosis and Management. www.nice.org.uk/guidance/ng115/resources/chronic-obstructive-pulmonarydisease-in-over-16s-diagnosis-and-management-pdf-66141600098245 Date last accessed: 29 April 2021. Date last updated: 5 December 2018.

21 Worth H, Buhl R, Criée CP, et al. The 'real-life' COPD patient in Germany: the DACCORD study. Respir Med 2016; 111: 64-71.

22 Graf J, Jörres RA, Lucke T, et al. Medical treatment of COPD: an analysis of guideline-adherent prescribing in a large national cohort (COSYCONET). Dtsch Arztebl Int 2018; 155: 599-605.

23 Steinmetz KO, Abenhardt B, Pabst S, et al. Assessment of physical functioning and handling of tiotropium/ olodaterol Respimat ${ }^{\oplus}$ in patients with COPD in a real-world clinical setting. Int J Chron Obstruct Pulmon Dis 2019; 14: 1441-1453.

24 Carone M, Pennisi A, D'Amato M, et al. Physical functioning in patients with chronic obstructive pulmonary disease treated with tiotropium/olodaterol Respimat in routine clinical practice in Italy. Pulm Ther 2020; 6: 261-274.

25 Miravitlles M, Montero-Caballero J, Richard F, et al. A cross-sectional study to assess inhalation device handling and patient satisfaction in COPD. Int J Chron Obstruct Pulmon Dis 2016; 11: 407-415.

26 Ställberg B, Nokela M, Ehrs PO, et al. Validation of the Clinical COPD Questionnaire (CCQ) in primary care. Health Qual Life Outcomes 2009; 7: 26.

27 van der Molen T, Willemse BW, Schokker S, et al. Development, validity and responsiveness of the Clinical COPD Questionnaire. Health Qual Life Outcomes 2003; 1: 13.

28 Zhou Z, Zhou A, Zhao Y, et al. Evaluating the Clinical COPD Questionnaire: a systematic review. Respirology 2017; 22: 251-262.

29 Valipour A, Tamm M, Kociánová J, et al. Improvement of self-reported physical functioning with tiotropium/ olodaterol in Central and Eastern European COPD patients. Int J Chron Obstruct Pulmon Dis 2019; 14: 2343-2354.

30 Worth H, Buhl R, Criée C-P, et al. GOLD 2017 treatment pathways in 'real life': an analysis of the DACCORD observational study. Respir Med 2017; 131: 77-84.

31 Ferguson GT, Buhl R, Bothner U, et al. Safety of tiotropium/olodaterol in chronic obstructive pulmonary disease: pooled analysis of three large, 52-week, randomized clinical trials. Respir Med 2018; 143: 67-73. 